Dimosthenis I. Bardzokas • D. V. Kushnir • L. A. Filshtinskii

\title{
Dynamic problems of the theory of elasticity for layers and semilayers with cavities
}

Received: 20 February 2008 / Revised: 3 November 2008 / Published online: 17 December 2008 (C) Springer-Verlag 2008

\begin{abstract}
We present a solution methodology for dynamic problems of the theory of elasticity based on the fundamental (F)-solutions approach for layers and semilayers containing cavities. Under the proposed solution framework boundary-value problems for three-dimensional cylindrical bodies are reduced to well-studied systems of one-dimensional singular integral equations. With the aid of the integral Fourier transform in time, we study the problem of impulse loading at the sides of cavities. We also demonstrate how the combination of the proposed methodology with the approach of reflections can be used for the solution of analogous problems for semi-infinite layers.
\end{abstract}

\section{Introduction}

Many contemporary problems of mechanics with both research and practical interest concern the study of the behavior of deformed bodies, which operate under the action of dynamic fields. There are two main difficulties in our ability to deal with such complex problems, namely (a) the one in formulating in a precise way the mathematical equations that approximate the behavior of the problem under study and (b) the one in solving efficiently the system of governing equations. Over the last 50 years, a vast number of approaches have been proposed toward this direction [1-35]. In this work, we focus on bodies, which are subject to both static and dynamic loads.

Generally speaking, mechanical structures and constructions are most often assembled from different elements, which usually have the shape of blocks or shells. When different kinds of heterogeneity, such as cracks, openings and inclusions are present, strong gradients of mechanical stresses appear which can lead to structural failure. Because of the importance of the applications, there has been an intense interest in investigating such phenomena. State-of-the-art numerical and analytical methods are engaged to improve the efficiency of the analysis. In particular, the solution of three-dimensional boundary problems of the theory of elasticity is sought by applying methods of the potential theory and uniform solutions, using superposition and eigenvector functions, integral equations, integral transforms, as well as direct numerical methods. Other approaches based on the theory of P-analytical functions and the general representations of the solutions are also important.

The method of uniform solutions, which was applied for the first time in [36,37] generates a system of partial solutions for three-dimensional problems of the theory of elasticity, which satisfy uniform conditions on flat surfaces of plates or on cylindrical borders of extended multiconnected cylinders. The basic system of uniform solutions is derived by exploiting the symbolic method of Lurie and Prokopov [36,37]. Vorovich and

D. I. Bardzokas $(\varangle)$

School of Applied Mathematics and Physical Sciences, National Technical University of Athens, 15773 Athens, Greece

E-mail: bardim@central.ntua.gr

D. V. Kushnir · L. A. Filshtinskii

Department of Applied Mathematics and Mechanics, Sumy State University, 40007 Sumy, Ukraine 
his collaborators have proposed a semi-inverse approach for the study of boundary problems for layers under the framework of elasticity, which in many cases simplifies the deduction of the solution [38-40].

Over the last years, the method of uniform solutions has been further advanced and generalized toward various directions. For example, Kosmodamiansky and Zhirov have studied problems of tension and bend of thick multiconnected piezo-passive and piezo-ceramic plates [41,48].

The methods of superposition and eigenvector functions, developed by Fridman, Grinchenko and Ulitko [49-51] have been used to determine partial solutions for different types of boundary surfaces. The methods of uniform solutions and eigenvector functions have been also used for the study of boundary-value problems of elasticity for finite cylinders or layers with cylindrical cavities. In turn, the method of superposition [51] is effective for domains with circular borders such as finite circular cylinders, layers with cavities of circular cross sections. On the other hand, the methods of potential theory [52-55] and integral transformations [56-58] are most-often used when the domain is bounded by contours of sufficiently arbitrary configuration [59-61].

Recently, the methods of uniform solutions have been used in combination with the approach of singular integral equations [62-75]. However, it is noteworthy to say that here the following two main difficulties arise: (a) a correspondence problem appears between the boundary conditions within the theory of elasticity and the boundary conditions for the enumerable set of meta-harmonic functions involved in the uniform solutions; (b) there is a need for normalization of the integrals that diverge on the boundary of the domain.

In this paper, we propose a method, which is based not on the uniform, but on the F-solutions approach for layers with cavities. The matrix of F-solutions is the Green matrix, which corresponds to the forces distributed along the segment: $x_{1}=x_{10}, x_{2}=x_{20},\left|x_{3}\right| \leq h$. With the aid of the derived $2 h$-periodic F-solutions, the boundary-value problems for three-dimensional cylindrical bodies are reduced to well-studied systems of one-dimensional singular integral equations having a relatively simple structure. We show how the integral Fourier transform in time can be used to examine the problem of impulse loading at the sides of cavities. Finally, we combine the proposed method with the method of reflections to solve analogous problems for semi-infinite layers. The paper is organized as follows. In the following section, we give the formal statement of the problem. In Sect. 3, we derive and discuss the so-called fundamental solution of the problem. In Sect. 4, we derive the integral equations governing the systems behavior. Section 5 focuses on the dynamic response of the systems under the action of pulse excitation. In Sect. 6, we present and discuss the simulation results and we conclude with Sect. 7.

\section{Statement of the problem}

In a Cartesian rectilinear coordinate system $O x_{1} x_{2} x_{3}$, let us examine an elastic uniform isotropic layer $-\infty<$ $x_{1}, x_{2}<\infty,\left|x_{3}\right| \leq h$, weakened by tunnel cavities along the axis $x_{3}$ with a common border of the cross section $\Gamma=\cup \Gamma_{v}\left(\cap \Gamma_{v}=\emptyset, v=1,2, \ldots, N\right)$. We assume that $\Gamma_{v}$ are the elementary closed contours without points of self-intersection with the continuous-according to Hölder-curvatures. On the surface of cavities $S=\cup S_{v}$, we define the vector of stresses $\left(X_{1 n}, X_{2 n}, X_{3 n}\right)(x, t), x=\left(x_{1}, x_{2}, x_{3}\right) \in S$. On the bases of the layer, the mixed-type uniform boundary conditions read:

$$
u_{1}=u_{2}=\sigma_{33}=0, \quad x_{3}= \pm h, \quad t>0 .
$$

The problem reduces to the determination of the wave field of the displacement vector $u=\left(u_{1}, u_{2}, u_{3}\right)$ and the stress tensor with components $\sigma_{i j}(i, j=1,2,3)$ with harmonic or pulse excitation of layers.

The wave field of displacements is determined by a system of Lamé equations

$$
\begin{aligned}
& \Delta u_{j}+\sigma \partial_{j} \vartheta+\frac{X_{j}}{\mu}=\frac{\rho}{\mu} \frac{\partial^{2} u_{j}}{\partial t^{2}} \quad j=1,2,3, \\
& \partial_{k}=\frac{\partial}{\partial x_{k}}, \quad \Delta=\partial_{k} \partial_{k}, \quad \vartheta=\partial_{k} u_{k}, \quad \sigma=\frac{1}{1-2 v},
\end{aligned}
$$

where $\Delta$ is the Laplace operator in $R^{3}, \vartheta$ is the three-dimensional expansion, $X_{j}$ is the intensity of the body forces, $\mu$ and $v$ are the module of shift and Poisson's coefficient, respectively, and $\rho$ is the density of the material.

Let us define

$$
c_{1}=\sqrt{\frac{\lambda+2 \mu}{\rho}}, \quad c_{2}=\sqrt{\frac{\mu}{\rho}}, \quad \gamma_{l}=\frac{\omega}{c_{l}}, \quad \mu_{l m}^{2}=\gamma_{l}^{2}-\lambda_{m}^{2}, \quad \Delta_{l m}=\partial_{1}^{2}+\partial_{2}^{2}+\mu_{l m}^{2} ; \quad l=1,2,
$$


where $c_{1}$ and $c_{2}$ are the velocities of propagation of longitudinal and transversal (shear) wave in the elastic body and $\gamma_{1}$ and $\gamma_{2}$ are the corresponding wave numbers.

We first examine the harmonic excitation of layer; let us assume

$$
u_{j}=\mathrm{e}^{-\mathrm{i} \omega t} U_{j}, \quad \vartheta=\mathrm{e}^{-\mathrm{i} \omega t} \theta=\mathrm{e}^{-\mathrm{i} \omega t} \partial_{k} U_{k}, \quad X_{j}=\mathrm{e}^{-\mathrm{i} \omega t} Y_{j}, \quad j=1,2,3
$$

where $U_{j}=U_{j}(x), \theta=\theta(x), Y_{j}=Y_{j}(x)\left(x=\left(x_{1}, x_{2}, x_{3}\right)\right)$ are the amplitudes of the corresponding magnitudes.

Eliminating time $t$ in Eq. (2) in accordance with the representations (3), we get the following system of differential equations:

$$
\Delta U_{j}+\sigma \partial_{j} \partial_{k} U_{k}+\gamma_{2}^{2} U_{j}=-\frac{Y_{j}}{\mu}, \quad j=1,2,3 .
$$

It is necessary to address the boundary conditions on the surfaces of the cavities reading:

$$
S_{i j} n_{i}=Y_{j n}, \quad j=1,2,3,
$$

where $S_{i j}$, and $Y_{j n}$ are the amplitude values of the magnitudes $\sigma_{i j}$ and $X_{j n}$, respectively.

Let us now examine the symmetrical state with respect to the median of the plane of the layer. The amplitudes of displacements and intensities of body forces can be represented in the form of Fourier series

$$
\begin{aligned}
\left\{U_{1}, U_{2}, \theta, Y_{1}, Y_{2}\right\} & =\sum_{m=1}^{\infty}\left\{U_{1 m}, U_{2 m}, \theta_{m} Y_{1 m}, Y_{2 m}\right\} \cos \lambda_{m} x_{3}, \\
\left\{U_{3}, Y_{3}\right\} & =\sum_{m=1}^{\infty}\left\{U_{3 m}, Y_{3 m}\right\} \sin \lambda_{m} x_{3}, \\
U_{j m} & =U_{j m}\left(x_{1}, x_{2}\right), \quad Y_{j m}=Y_{j m}\left(x_{1}, x_{2}\right) ; \quad j=1,2,3, \\
\theta_{m} & =\partial_{1} U_{1 m}+\partial_{2} U_{2 m}+\lambda_{m} U_{3 m}, \quad \lambda_{m}=\pi(2 m-1) /(2 h) .
\end{aligned}
$$

In this case, the boundary conditions (1) on the bases of the layer are satisfied.

Eliminating the thickness coordinate $x_{3}$ in Eqs. (4) and with the aid of the representations (6) we get the following system of equations with respect to the Fourier coefficients $U_{j m}$ :

$$
\begin{gathered}
\Delta_{2 m} U_{l m}+\sigma \partial_{l} \theta_{m}=-\frac{Y_{l m}}{\mu}, \quad l=1,2, \\
\Delta_{2 m} U_{3 m}-\sigma \lambda_{m} \theta_{m}=-\frac{Y_{3 m}}{\mu} ; \quad m=1,2, \ldots
\end{gathered}
$$

To eliminate the thickness coordinate from Eq. (5) we use the following representations for the amplitudes of the components of the stress tensor and the vector of surface load:

$$
\begin{aligned}
\left\{S_{l k}, Y_{i n}\right\} & =\sum_{m=1}^{\infty}\left\{S_{l k}^{(m)}, Y_{i n}^{(m)}\right\} \cos \lambda_{m} x_{3}, \quad l, k=1,2, \\
\left\{S_{j 3}, Y_{3 n}\right\} & =\sum_{m=1}^{\infty}\left\{S_{j 3}^{(m)}, Y_{3 n}^{(m)}\right\} \sin \lambda_{m} x_{3}, \quad j=1,2,3,
\end{aligned}
$$

Then, the boundary conditions (5) are split to a set of equalities of the form

$$
S_{i j}^{(m)} n_{i}=Y_{j n}^{(m)}, \quad j=1,2,3 ; m=1,2, \ldots
$$

Thus, the problem is reduced to the solution of the system of differential equations (7) with boundary conditions (9) for every specific value of $m$. 


\section{Fundamental (F) solutions for the layer}

Lets us now assume that distributed forces with linear intensities $\left\{P_{1}, P_{2}, P_{3}\right\}\left(x_{3}\right)$ act along the cord $x_{1}=0$, $x_{2}=0,\left|x_{3}\right| \leq h$. Then the Fourier coefficients of intensities of the body forces appearing on the right side of Eqs. (7) take the form

$$
Y_{j m}=P_{j m} \delta(x), \quad x=\left(x_{1}, x_{2}\right), \quad j=1,2,3
$$

where $\delta(x)$ is the two-dimensional delta function.

The F-solutions for the layer, which correspond to the mixed boundary conditions (1) are given by the components of the matrix of the fundamental solutions of the system (7) with the right-hand sides, determined by relations (10). The derivation procedure of the F-solutions is described in detail in Appendix A.

The true displacement values for the general case can be determined by the formulas

$$
\begin{aligned}
u_{l} & =\operatorname{Re}\left(\mathrm{e}^{-\mathrm{i} \omega t} \sum_{j=1}^{3} \sum_{m=1}^{\infty} U_{l m}^{(j)} \cos \lambda_{m} x_{3}\right), \quad l=1,2 ; \\
u_{3} & =\operatorname{Re}\left(\mathrm{e}^{-\mathrm{i} \omega t} \sum_{j=1}^{3} \sum_{m=1}^{\infty} U_{3 m}^{(j)} \sin \lambda_{m} x_{3}\right), \\
U_{n m}^{(j)} & =\frac{i P_{j m}}{4 \mu} g_{n m}^{(j)}
\end{aligned}
$$

where $g_{n m}^{(j)}$ are the components of the matrix of F-solutions for every fixed value $m$.

The expressions (11), (A7) give a representation of the waveguide properties of the layer. It is obvious that for any frequency of excitation we can always find a number $m$, for which the characteristic number $\mu_{1 m}$ or both characteristic numbers become pure imaginary. This leads to nonuniform, exponentially damped-along $r$-waves. Whenever $\pi(2 m-1)<2 \gamma_{1} h$, the first $m$ terms in series (11) represent the superposition of the waves spread from the source. The terms of the series that satisfy the inequality $\pi(2 m-1)>2 \gamma_{2} h$ damp exponentially with the increase of $r$ and the increase of number $m$. It follows that when the waveguide gets thicker, the spectrum of frequencies, which are allowed to pass, gets wider.

From the above, it is obvious that the remainders of the series (11) converge to zero, since the general term of any of these series exponentially decreases with the increase of number $m$. It is possible to show that these series converge absolutely, whenever $r \neq 0$.

\section{The boundary problem}

\subsection{Integral representation of the solutions}

Assume that $f \in C^{2}(\bar{G})$, where $G=R^{2} \backslash \Gamma$ is the physical field with line of disruption $\Gamma$. Let us write the formulas for the generalized derivatives,

$$
\partial_{j} f=\left\{\partial_{j} f\right\}+n_{j}[f] \delta_{\Gamma}, \quad\left(\partial_{1}^{2}+\partial_{2}^{2}\right) f=\left\{\left(\partial_{1}^{2}+\partial_{2}^{2}\right) f\right\}+\left[\frac{\partial f}{\partial n}\right] \delta_{\Gamma}+\frac{\partial}{\partial n}\left([f] \delta_{\Gamma}\right),
$$

where $\{\cdot\}$ is the corresponding classical derivative, $[\cdot]$ is the jump of the indicated function on the contour $\Gamma, n_{j}$ is the projection of the unit vector of normal to the contour $\Gamma$ on the axis $x_{j} ;[\cdot] \delta_{\Gamma}$ and $\frac{\partial}{\partial n}\left([\cdot] \delta_{\Gamma}\right)$ are the simple and the dual layer, respectively [76].

Introducing these relations into (7), we can represent it in the form:

$$
\begin{aligned}
& \Delta_{2 m} U_{j m}+\sigma \partial_{j} \theta_{m}=f_{j m}, \quad j=1,2,3 ; \quad m=1,2, \ldots \\
& f_{j m}=-\left[\frac{\partial U_{j m}}{\partial n}\right] \delta_{\Gamma}-\frac{\partial}{\partial n}\left(\left[U_{j m}\right] \delta_{\Gamma}\right)-\sigma\left[\theta_{m}\right] n_{j} \delta_{\Gamma}, \quad n_{3}=0,
\end{aligned}
$$


Using the matrix of the F-solutions (A7), the solution of the system (12) can be represented in the form of convolution

$$
U_{m}(x)=\left\{U_{1 m}, U_{2 m}, U_{3 m}\right\}=g_{m} * f_{m} ; \quad x=\left(x_{1}, x_{2}\right), \quad f_{m}=\left\{f_{1 m}, f_{2 m}, f_{3 m}\right\} .
$$

Hence we obtain in an expanded form the integral representations of the wave field of displacements (in the sequel, integration is conducted along the contour $\Gamma$, whenever nothing else is explicitly stipulated)

$$
\begin{aligned}
U_{j m}(x)= & \int\left[U_{k m}\right](y) \frac{\partial}{\partial n_{y}} g_{j m}^{(k)}(x-y) \mathrm{d} S_{y} \\
& -\int\left(\left[\frac{\partial U_{k m}}{\partial n}\right]+\sigma\left[\theta_{m}\right] n_{k}\right)(y) g_{j m}^{(k)}(x-y) \mathrm{d} S_{y}, \quad j=1,2,3 ; \quad m=1,2, \ldots,
\end{aligned}
$$

where $\mathrm{d} S_{y}$ is an element of arc of the contour $\Gamma$ and summing is conducted on $k=1,2,3$.

Whenever the contour $\Gamma$ is a set of nonintersected arcs (mathematical sections) $\Gamma_{v}(v=1,2, \ldots, N)$ and the stress vector is continuously extendable through all $\Gamma_{\nu}$, it is sufficient to leave the first term (addend) in the right-hand side of (13), i.e., to seek the solution in the form of generalized potentials of dual layer. For the solution of the above problem, we leave only the second term (addend) and seek for the solution in the form of generalized potentials of single layers, which, in expanded form, read:

$$
\begin{aligned}
U_{1 m}(z) & =\frac{1}{\gamma_{2}^{2}} \int\left[\frac{\partial}{\partial \xi_{1}}\left(-p_{m} \frac{\partial}{\partial \xi_{1}}-q_{m} \frac{\partial}{\partial \xi_{2}}+r_{m} \lambda_{m}\right) H(r)+p_{m} \gamma_{2}^{2} H_{0}^{(1)}\left(\mu_{2 m} r\right)\right] \mathrm{d} S, \\
U_{2 m}(z) & =\frac{1}{\gamma_{2}^{2}} \int\left[\frac{\partial}{\partial \xi_{2}}\left(-p_{m} \frac{\partial}{\partial \xi_{1}}-q_{m} \frac{\partial}{\partial \xi_{2}}+r_{m} \lambda_{m}\right) H(r)+q_{m} \gamma_{2}^{2} H_{0}^{(1)}\left(\mu_{2 m} r\right)\right] \mathrm{d} S, \\
U_{3 m}(z) & =\frac{1}{\gamma_{2}^{2}} \int\left[\lambda_{m}\left(-p_{m} \frac{\partial}{\partial \xi_{1}}-q_{m} \frac{\partial}{\partial \xi_{2}}+r_{m} \lambda_{m}\right) H(r)+r_{m} \gamma_{2}^{2} H_{0}^{(1)}\left(\mu_{2 m} r\right)\right] \mathrm{d} S, \\
\theta_{m}(z) & =\int\left(-p_{m} \frac{\partial}{\partial \xi_{1}}-q_{m} \frac{\partial}{\partial \xi_{2}}+r_{m} \lambda_{m}\right) H_{0}^{(1)}\left(\mu_{1 m} r\right) \frac{\mathrm{d} S}{1+\sigma},
\end{aligned}
$$

where $p_{m}=\left\{p_{m}^{v}(\zeta), \zeta \in \Gamma_{\nu}\right\}, q_{m}=\left\{q_{m}^{v}(\zeta), \zeta \in \Gamma_{\nu}\right\}, r_{m}=\left\{r_{m}^{v}(\zeta), \zeta \in \Gamma_{v}\right\} ; \zeta=\xi_{1}+\mathrm{i} \xi_{2} \in \Gamma=\cup \Gamma_{\nu}, \mathrm{d} S$ is the element of the arc of contour $\Gamma, \zeta-z=r \mathrm{e}^{\mathrm{i} \alpha} ; H_{p}^{(1)}(x)$ are the Hankel functions of first order of the degree $p, H(r)=H_{0}^{(1)}\left(\mu_{1 m} r\right)-H_{0}^{(1)}\left(\mu_{2 m} r\right)$.

\subsection{The system of integral equations of boundary problems}

At this point, we represent the boundary conditions (9) on $\Gamma$ in a complex form:

$$
\begin{aligned}
& S_{1}^{(m)}-\mathrm{e}^{2 \mathrm{i} \psi} S_{2}^{(m)}=2 \mathrm{e}^{\mathrm{i} \psi}\left(Y_{1}^{(m)}-\mathrm{i} Y_{2}^{(m)}\right)=2\left(N^{(m)}-\mathrm{i} T^{(m)}\right) \\
& S_{1}^{(m)}-\mathrm{e}^{-2 \mathrm{i} \psi} \tilde{S}_{2}^{(m)}=2 \mathrm{e}^{\mathrm{i} \psi}\left(Y_{1}^{(m)}+\mathrm{i} Y_{2}^{(m)}\right)=2\left(N^{(m)}+\mathrm{i} T^{(m)}\right) \\
& \mathrm{e}^{\mathrm{i} \psi} S_{3}^{(m)}+\mathrm{e}^{-\mathrm{i} \psi} \tilde{S}_{3}^{(m)}=2 Y_{3}^{(m)}, \quad(m=1,2, \ldots) \\
& S_{1}^{(m)}=S_{11}^{(m)}+S_{22}^{(m)}, \\
& S_{2}^{(m)}=S_{22}^{(m)}-S_{11}^{(m)}+2 \mathrm{i} S_{12}^{(m)}, \quad \tilde{S}_{2}^{(m)}=S_{22}^{(m)}-S_{11}^{(m)}-2 \mathrm{i} S_{12}^{(m)}, \\
& S_{3}^{(m)}=S_{13}^{(m)}-\mathrm{i} S_{23}^{(m)}, \quad \tilde{S}_{3}^{(m)}=S_{13}^{(m)}+\mathrm{i} S_{23}^{(m)},
\end{aligned}
$$

where $\psi$ is the angle between the normal to the contour $\Gamma$ and the axis $O x_{1}, N^{(m)}$ and $T^{(m)}$ are the Fourier coefficients of the amplitudes of the normal and tangent stresses on $\Gamma$. 
Using Hooke's law in amplitudes, we obtain the representations of the combinations introduced in (15) in terms of the components of the displacement vector

$$
\begin{aligned}
& S_{1}^{(m)}=2 \mu\left(\sigma \theta_{m}-\lambda_{m} U_{3 m}\right), \\
& S_{2}^{(m)}=-4 \mu \frac{\partial}{\partial z}\left(U_{1 m}-\mathrm{i} U_{2 m}\right), \quad \tilde{S}_{2}^{(m)}=-4 \mu \frac{\partial}{\partial \bar{z}}\left(U_{1 m}+\mathrm{i} U_{2 m}\right), \\
& S_{3}^{(m)}=\mu\left\{2 \frac{\partial}{\partial z} U_{3 m}-\lambda_{m}\left(U_{1 m}-\mathrm{i} U_{2 m}\right)\right\}, \quad \frac{\partial}{\partial z}=\frac{1}{2}\left(\partial_{1}-\mathrm{i} \partial_{2}\right), \\
& \tilde{S}_{3}^{(m)}=\mu\left\{2 \frac{\partial}{\partial \bar{z}} U_{3 m}-\lambda_{m}\left(U_{1 m}+\mathrm{i} U_{2 m}\right)\right\}, \quad \frac{\partial}{\partial \bar{z}}=\frac{1}{2}\left(\partial_{1}+\mathrm{i} \partial_{2}\right) .
\end{aligned}
$$

Let us introduce the functions $y_{j m}$ by the equalities

$$
p_{m}=y_{1 m} \mathrm{e}^{\mathrm{i} \psi}+y_{2 m} \mathrm{e}^{-\mathrm{i} \psi}, \quad q_{m}=\mathrm{i}\left(y_{2 m} \mathrm{e}^{-\mathrm{i} \psi}-y_{1 m} \mathrm{e}^{\mathrm{i} \psi}\right), \quad r_{m}=y_{3 m}
$$

Substituting the limiting values of the combinations (16) into the boundary equalities (15) with the use of the representations (14) and taking into account the formulas (17), we obtain the following system of singular integral equations of the boundary-value problem (7), (9):

$$
\begin{aligned}
& \mp \mathrm{i} y_{1 m}\left(\zeta_{0}\right)+\frac{1}{4} \int_{\Gamma}\left[y_{1 m}(\zeta) K_{11}+y_{2 m}(\zeta) K_{12}+y_{3 m}(\zeta) K_{13}\right] \mathrm{d} S=\frac{\left(Y_{1}^{(m)}+\mathrm{i} Y_{2}^{(m)}\right) \mathrm{e}^{-\mathrm{i} \psi_{0}}}{4 \mu}, \\
& \mp \mathrm{i} y_{2 m}\left(\zeta_{0}\right)+\frac{1}{4} \int_{\Gamma}\left[y_{1 m}(\zeta) K_{21}+y_{2 m}(\zeta) K_{22}+y_{3 m}(\zeta) K_{23}\right] \mathrm{d} S=\frac{\left(Y_{1}^{(m)}-\mathrm{i} Y_{2}^{(m)}\right) \mathrm{e}^{\mathrm{i} \psi_{0}}}{4 \mu}, \\
& \mp \mathrm{i} y_{3 m}\left(\zeta_{0}\right)+\frac{1}{4} \int_{\Gamma}\left[y_{1 m}(\zeta) K_{31}+y_{2 m}(\zeta) K_{32}+y_{3 m}(\zeta) K_{33}\right] \mathrm{d} S=\frac{Y_{3}^{(m)}}{2 \mu} .
\end{aligned}
$$

Here $K_{i j}(i, j=1,2,3)$ are the singular kernels, extracted in Appendix C.

The sum-total index of system (18) equals to zero; therefore, it is uniquely solvable for any frequency $\omega$, which does not belong to the spectrum.

The system (18) can be used both for the investigation of the fluctuations of thick plates having weakened cavities and for the investigation of wave fields in thick-walled cylinders. In the latter case, the upper sign with the term outside the integrals corresponds to the external side of the cylinders, and the lower sign to the internal side.

Let us now express the normal stress $\sigma_{\theta \theta}$ on the bounding surfaces $\Gamma_{\nu}$. Using the relations (14) and (16), we can represent it in the form

$$
\begin{aligned}
\sigma_{\theta \theta}= & \left|S_{\theta \theta}\right| \cos (\omega t-\Omega), \quad \Omega=-\arg S_{\theta \theta}, \quad S_{\theta \theta}=\sum_{m=1}^{\infty} S_{\theta \theta}^{(m)} \cos \lambda_{m} x_{3}, \\
S_{\theta \theta}^{(m)}= & S_{1}^{(m)}-N^{(m)}=\mp \frac{\mathrm{i}\left(y_{1 m}\left(\zeta_{0}\right)+y_{2 m}\left(\zeta_{0}\right)\right)}{1-v} \\
& +\int\left\{\left(y_{1 m}(\zeta) \mathrm{e}^{\mathrm{i}\left(\psi-\alpha_{0}\right)}+y_{2 m}(\zeta) \mathrm{e}^{\mathrm{i}\left(\alpha_{0}-\psi\right)}\right)\left(\frac{\mu_{1 m}}{2(1-v)} H_{11 m}^{0}-\lambda_{m} g_{1 m}\right)\right. \\
& \left.+y_{3 m}\left(\zeta_{0}\right) \lambda_{m}\left[\frac{1}{2(1-v)} H_{01 m}^{0}-H_{02 m}^{0}-\frac{\lambda_{m}^{2}}{\gamma_{2}^{2}} H^{0}\right]\right\} \mathrm{d} S-N^{(m)}, \quad \zeta_{0} \in \Gamma .
\end{aligned}
$$




\section{The nonstationary problem}

\subsection{Pulse excitation of layers}

In this section, we examine the problem of the pulse excitation of layers through the lateral surface of transparent tunnel cavities. Introducing the integral Fourier transform in time, one obtain

$$
\begin{aligned}
& U_{j}(x, \omega) \frac{1}{\sqrt{2 \pi}} \int_{0}^{\infty} u_{j}(x, t) \mathrm{e}^{\mathrm{i} \omega t} \mathrm{~d} t,\left.\quad u_{j}\right|_{t=0}=\left.\frac{\partial u_{j}}{\partial t}\right|_{t=0}=0, \\
& u_{j}(x, t)=\sqrt{\frac{2}{\pi}} \operatorname{Re} \int_{0}^{\infty} U_{j}(x, \omega) \mathrm{e}^{-\mathrm{i} \omega t} \mathrm{~d} \omega ; \quad j=1,2,3 .
\end{aligned}
$$

Actually, the problem now is reduced to an analysis of the boundary problem given by Eqs. (7)-(9) with respect to the Fourier transformation of the corresponding system of integral equations (18), where the right-hand sides represent the spectral functions acting on the surface of the cavity. The solution of the pulse problem can now be obtained by the superposition of the "elementary" solutions on the entire spectrum of frequencies.

5.2 The Boundary-value problem for semi-infinite layers

In a Cartesian rectilinear coordinate system $O x_{1} x_{2} x_{3}$, we consider the semilayer $-\infty<x_{1}<+\infty, 0<x_{2}<$ $+\infty,\left|x_{3}\right| \leq h$, weakened by a transparent tunnel cavity with a border of a cross section $\Gamma$. We assume that $\Gamma \cap R_{1}=\varnothing$ and on the bases of the semilayer the boundary conditions given by Eq. (1) hold.

On the border of the semilayer, two types of boundary conditions are examined:

$$
\begin{aligned}
& \text { (a) } u_{1}=u_{3}=\sigma_{22}=0, \quad x_{2}=0, \\
& \text { (b) } \sigma_{13}=\sigma_{23}=u_{2}=0, \quad x_{2}=0 .
\end{aligned}
$$

Here, we examine the problem of the pulse excitation of semilayers through the lateral surface of a cavity. We use the proposed approach, described in Sect. 5.1, in combination with the method of reflections. In this case, the matrix of F-solutions is written in the form of $g_{m}+A g_{m}^{*}$, where $g_{m}$ is the matrix of F-solutions for the layer (A7) and $g_{m}^{*}$ is the matrix corresponding to the conjugate source. The values of $A=-1, A=1$ and $A=0$ correspond to the semilayer with boundary conditions given by (20), (21) and the one with tunnel cavity, respectively.

\section{Numerical results}

The calculations are presented in the following sequence. First, we find the approximate numerical solution of the system of integral equations (18) by the method of mechanical quadratures, and then we derive with their help the amplitude values of the mechanical stresses and displacements. The numerical scheme is described in detail in Appendix C.

\subsection{Harmonic excitation of layers or finite cylinders}

Let there be a layer weakened by a tunnel cavity with a contour of an cross section in the form of an ellipsis $\left(\zeta=R_{1} \cos \phi+\mathrm{i} R_{2} \sin \phi\right)$ or square with filleted corners $\left(\zeta=R\left(\mathrm{e}^{\mathrm{i} \phi}+0.14036 \mathrm{e}^{-3 \mathrm{i} \phi}\right)\right)$. On the surface of the cavity—harmonically oscillating in time—normal pressure acts

$$
N=N_{0} \cos \frac{\pi x_{3}}{2 h}, \quad N_{0}=\text { const },
$$

where $N$ is the amplitude of oscillation.

Let us now examine the layer, weakened by two tunnel cavities- $\operatorname{circular}(R=1)$ and elliptical $\left(R_{1}=1\right.$, $\left.R_{2}=1.5\right)$. 


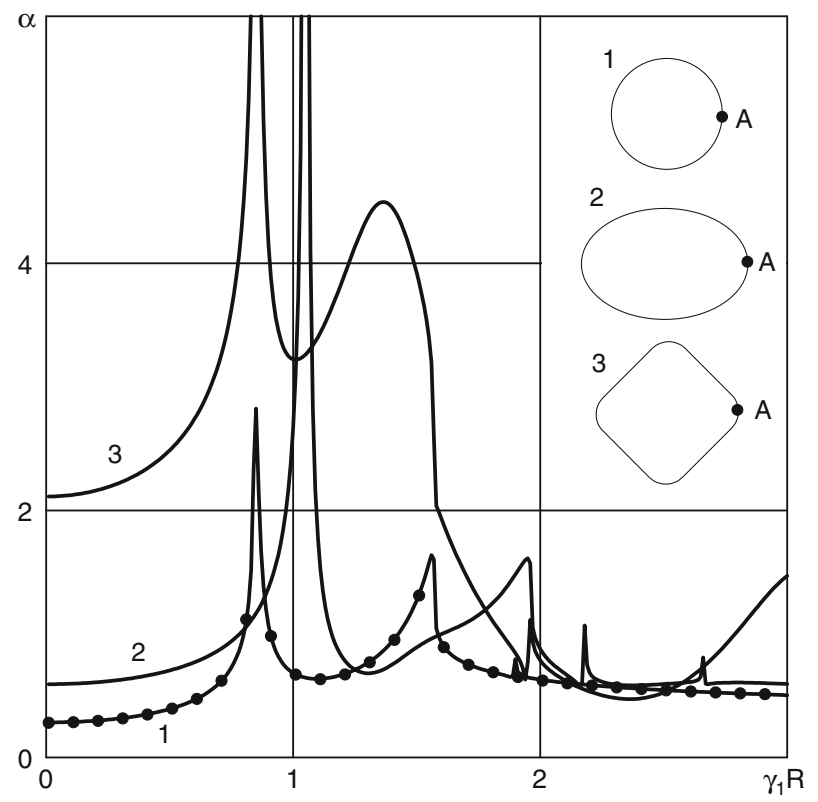

Fig. 1 The change of the relative magnitude $\alpha=\left|S_{\theta \theta} / N_{0}\right|$ at the point $A$ on the axis $O x_{1}\left(x_{3}=0\right)$, depending on the relative wave number $\gamma_{1} R$ for the cavity of circular $\left(R_{1}=R_{2}=R=1\right)$, elliptical $\left(R_{1}=1.5, R_{2}=1, R=\left(R_{1}+R_{2}\right) / 2\right)$ and square $(R=1)$ cut

Figure 1 depicts the change of the relative magnitude $\alpha=\left|S_{\theta \theta} / N_{0}\right|$ at the point $A$ on the axis $O x_{1}\left(x_{3}=0\right)$ with respect to the wave number $\gamma_{1} R$ for the cavity of "circular" $\left(R_{1}=R_{2}=R=1\right)$, "elliptical" $\left(R_{1}=1.5, R_{2}=1, R=\left(R_{1}+R_{2}\right) / 2\right)$ and "square" $(R=1)$ cut. For our simulations, we used the flowing values of $h=1$ and $v=0.3$. The Curves 1,2 and 3 are constructed for the circle, the ellipsis and the square, respectively. The points on the figure indicate the exact solution, which, for the circular cut, was obtained in series.

The curves on Fig. 2 illustrate the distribution of the magnitude $\alpha$ along the contour of the circular opening (in the median plane of layer) with a cross connection between them. $d=1$ is for the Curve $1, d=3$ for the Curve 2 and $d=5$ for the Curve 3. In all cases, there is a normal pressure with amplitude given by Eq. (22) acting on the surface of the circular cavity. The remaining parameters have the same values as above.

Figure 3 depicts the amplitude-frequency characteristics of the magnitude $\alpha$ for a hollow concentric cylinder, whose internal contour of cross section is a circle of radius $R=1$, the outer contour is a square with filleted corners, whose parametric equation has the form $\zeta=R_{2}\left(\mathrm{e}^{\mathrm{i} \phi}+0.14036 \mathrm{e}^{-3 \mathrm{i} \phi}\right), 0 \leq \phi \leq 2 \pi, R_{2}=1.5$.

On the internal surface acts a normal pressure with amplitude (22). The calculation is conducted at the point $A$ with coordinates $(1,0,0)$.

\subsection{Pulse excitation of layers}

Let there be an impulse-like pressure ( $T$ is the impulse length) acting on the surface of the circular cavity in the following manner:

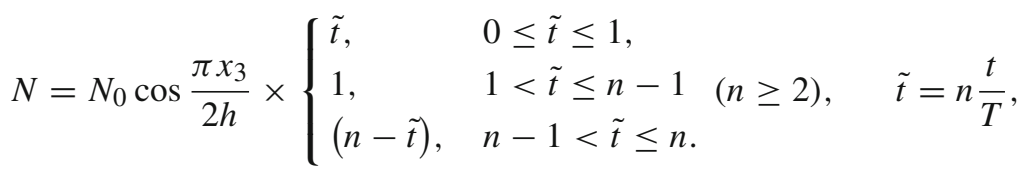

Figure 4 shows the results of the calculations for the evolution of circumferential normal stress $\sigma_{\theta \theta}$ in time for different values of the half-thickness of layer $h$. For our simulations, we used $T=0.1, x_{3}=0$, $R=10, v=0.3, n=5$, while the velocity of propagation of the longitudinal wave was set to $c_{2}=5850 \mathrm{M} / \mathrm{C}$. 


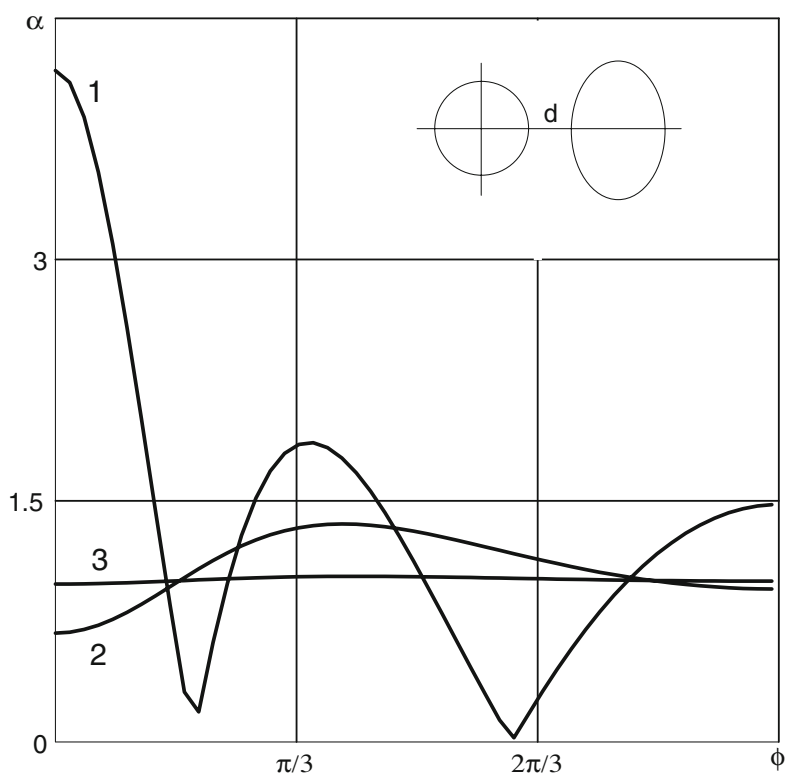

Fig. 2 The distribution of the magnitude $\alpha$ along the contour of the circular opening (in the median plane of layer) with cross connection between them. $d=1$ is for the Curve $1, d=3$ for the Curve 2 and $d=5$ for the Curve 3

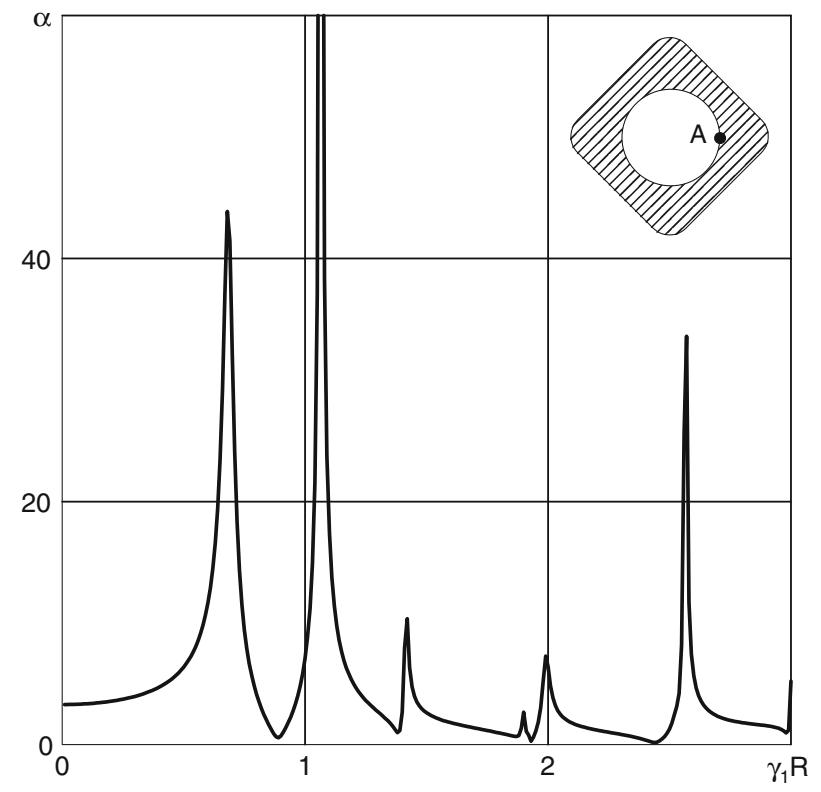

Fig. 3 The amplitude-frequency characteristics of the magnitude $\alpha$ for a hollow concentric cylinder

We also examined the problem of a semilayer $-\infty<x_{1}<+\infty, 0<x_{2}<+\infty,\left|x_{3}\right| \leq h$, weakened by a transparent tunnel cavity $\zeta=R \mathrm{e}^{\mathrm{i} \phi}+z_{1}$. We assumed that a pulse acts on the surface of cavity (23). Figure 5 shows the change of the magnitude $-\mu u_{2}$ at the point $z_{0}=(0,150)$ in the median plane of the layer when $z_{1}=(0,200), R=10, v=0.3, h=100, c_{2}=5850$. Here, the solid line corresponds to the infinite layer, the dashed line to the semilayer with the boundary conditions given as in Eq. (20) and the dotted one to the semilayer with the boundary conditions given as in Eq. (21). Point $A$ on the graph indicates the moment of time when the straight wave reaches point $z_{0}$. Point $B$ is the time of arrival at the point $z_{0}$ of the wave, reflected from the border of the semilayer. 


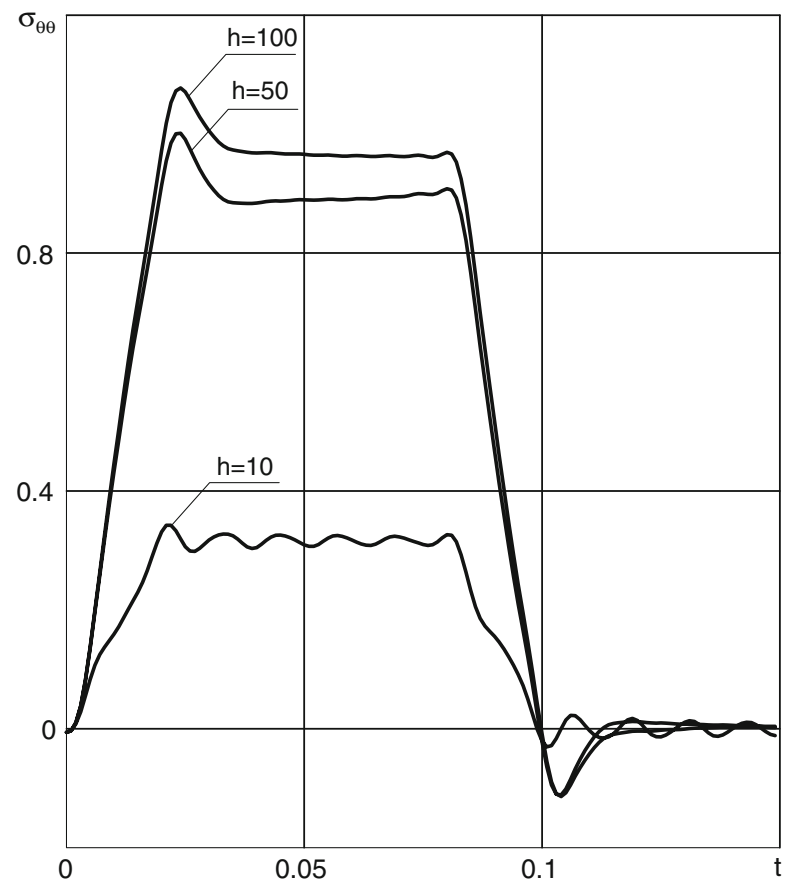

Fig. 4 The evolution of circumferential normal stress $\sigma_{\theta \theta}$ in time for different values of the half-thickness of layer $h$

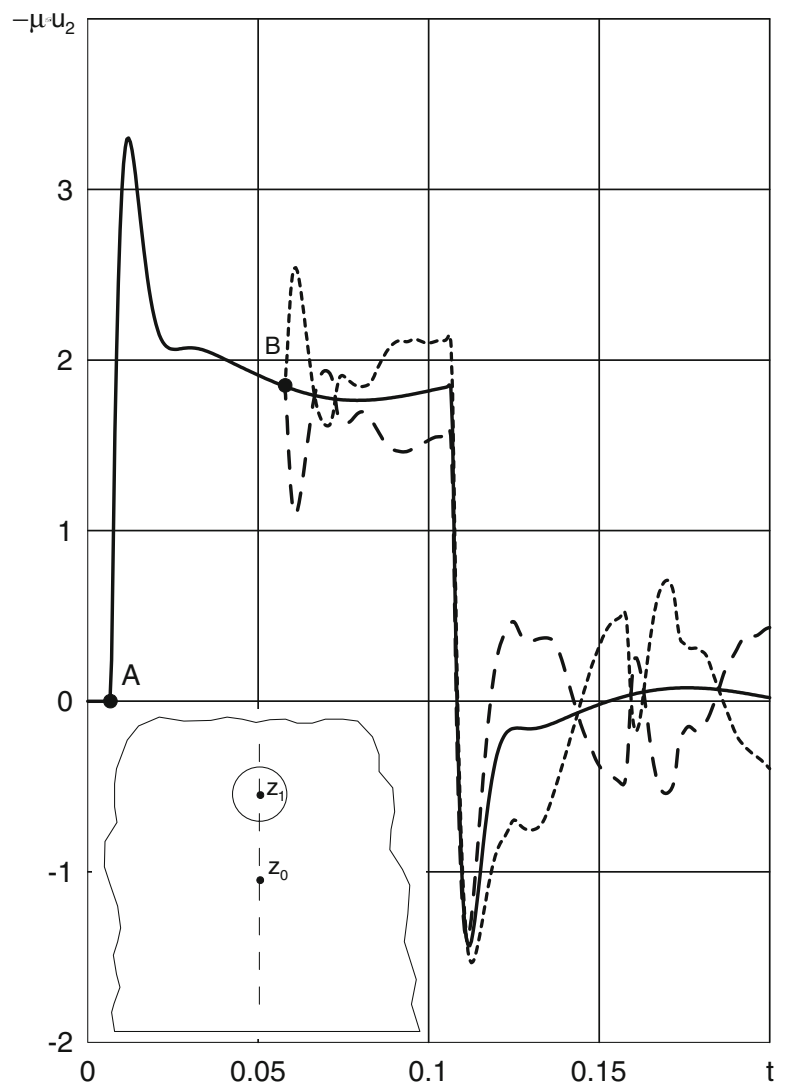

Fig. 5 The change of the magnitude $-\mu u_{2}$ at the point $z_{0}=(0,150)$ in the median plane of the layer when $z_{1}=(0,200)$, $R=10, v=0.3, h=100, c_{2}=5850$. The solid line corresponds to the infinite layer, the dashed one to the semilayer with the boundary conditions (5.1) and the dotted line to the semilayer with the boundary conditions (5.2) 


\section{Conclusions}

In this work, we further developed the fundamental-solutions approach for layers. We used the proposed methodology to examine several problems of harmonic and impulse oscillations. We derived the amplitude characteristics as well as the stress diagrams for layers with one or two cavities of different types. We also examined the spectrum of finite cylinders with thick walls. With the aid of Fourier integral transformation, we solved problems for impulse loading on layers and semilayers with cavities. The results of this work can be used to study the strength of constructions in the form of finite cylinders and thick layers with cavities, problems most often met in the field of rock-mechanics.

\section{Appendices}

\section{A The procedure for obtaining F-solutions}

From system (7), taking into account Eq. (10), we obtain

$$
\begin{aligned}
\Delta_{2 m} U_{1 m}+\sigma \partial_{1} \theta_{m} & =-\frac{P_{1 m}}{\mu} \delta(x), \\
\Delta_{2 m} U_{2 m}+\sigma \partial_{2} \theta_{m} & =-\frac{P_{2 m}}{\mu} \delta(x), \\
\Delta_{2 m} U_{3 m}-\sigma \lambda_{m} \theta_{m} & =-\frac{P_{3 m}}{\mu} \delta(x) ; \quad m=1,2, \ldots
\end{aligned}
$$

Differentiating each one of the equations of system (A1) with respect to the variable $x_{j}$ and summing up, we obtain the following equation with respect to $\theta_{m}$ :

$$
\Delta_{1 m} \theta_{m}=-\frac{1}{\mu(1+\sigma)}\left(P_{1 m} \partial_{1}+P_{2 m} \partial_{2}+P_{3 m} \lambda_{m}\right) \delta(x)
$$

Let us now examine the case of $P_{1} \neq 0, P_{2}=P_{3}=0$ in detail.

From Eq. (A2), for the considered case, we obtain the nonhomogeneous Helmholtz equation

$$
\Delta_{1 m} \theta_{m}^{(1)}=-\frac{P_{1 m}}{\mu(1+\sigma)} \partial_{1} \delta(x)
$$

Let $E$ be the fundamental solution of Helmholtz operator. Taking into account that the function $\delta(x)$ is finite and that the convolution $E * \partial_{1} f=f * \partial_{1} E$ exists, we obtain from Eq. (A3)

$$
\theta_{m}^{(1)}=\frac{\mathrm{i} P_{1 m}}{4 \mu(1+\sigma)} \partial_{1} H_{0}^{(1)}\left(\mu_{1 m} r\right)
$$

where $H_{p}^{(1)}(x)$ is the Hankel function of the first order, of degree $p$. (A4) enables us to split the equations in system (A1) and represent it in the form:

$$
\begin{aligned}
\Delta_{2 m} U_{1 m}^{(1)} & =-\frac{\mathrm{i} \sigma P_{1 m}}{4 \mu(1+\sigma)} \partial_{1}^{2} H_{0}^{(1)}\left(\mu_{1 m} r\right)-\frac{P_{1 m}}{\mu} \delta(x), \\
\Delta_{2 m} U_{2 m}^{(1)} & =-\frac{\mathrm{i} \sigma P_{1 m}}{4 \mu(1+\sigma)} \partial_{1} \partial_{2} H_{0}^{(1)}\left(\mu_{1 m} r\right), \\
\Delta_{2 m} U_{3 m}^{(1)} & =\frac{\mathrm{i} \sigma P_{1 m}}{4 \mu(1+\sigma)} \lambda_{m} \partial_{1} H_{0}^{(1)}\left(\mu_{1 m} r\right) .
\end{aligned}
$$


Integration of the system (A5) in the space of generalized functions $\mathcal{D}^{\prime}\left(R^{2}\right)$ gives

$$
\begin{aligned}
U_{1 m}^{(1)} & =\frac{\mathrm{i} P_{1 m}}{4 \mu}\left(-\frac{1}{\gamma_{2}^{2}} \partial_{1}^{2} H(r)+H_{0}^{(1)}\left(\mu_{2 m} r\right)\right), \\
U_{2 m}^{(1)} & =-\frac{\mathrm{i} P_{1 m}}{4 \mu \gamma_{2}^{2}} \partial_{1} \partial_{2} H(r), \\
U_{3 m}^{(1)} & =\frac{\mathrm{i} P_{1 m}}{4 \mu \gamma_{2}^{2}} \partial_{1} \lambda_{m} H(r) ; \quad H(r)=H_{0}^{(1)}\left(\mu_{1 m} r\right)-H_{0}^{(1)}\left(\mu_{2 m} r\right) .
\end{aligned}
$$

The cases when $P_{2} \neq 0, P_{1}=P_{3}=0$ and $P_{3} \neq 0, P_{1}=P_{2}=0$ are examined in an analogous manner. Let us extract the final results for the displacement vector

$$
U_{n m}^{(j)}=\frac{\mathrm{i} P_{j m}}{4 \mu} g_{n m}^{(j)} .
$$

The magnitudes $g_{n m}^{(j)}$ are components of the matrix of F-solutions for every fixed value $m$ :

$$
\begin{aligned}
& g_{m}=\left\|g_{n m}^{(j)}\right\|, \quad n, j=1,2,3 ; m=1,2, \ldots \\
& g_{1 m}^{(1)}=\frac{1}{2 \gamma_{2}^{2}} \sum_{l=1}^{2}(-1)^{l+1} \mu_{l m}^{2}\left(H_{0}^{(1)}\left(\mu_{l m} r\right)-H_{2}^{(1)}\left(\mu_{l m} r\right) \cos 2 \alpha\right)+H_{0}^{(1)}\left(\mu_{2 m} r\right), \\
& g_{2 m}^{(1)}=g_{1 m}^{(2)}=-\frac{1}{2 \gamma_{2}^{2}} \sum_{l=1}^{2}(-1)^{l+1} \mu_{l m}^{2} H_{2}^{(1)}\left(\mu_{l m} r\right) \sin 2 \alpha, \\
& g_{3 m}^{(1)}=-g_{1 m}^{(3)}=-\frac{\lambda_{m}}{\gamma_{2}^{2}} \sum_{l=1}^{2}(-1)^{l+1} \mu_{l m} H_{1}^{(1)}\left(\mu_{l m} r\right) \cos \alpha, \\
& g_{2 m}^{(2)}=\frac{1}{2 \gamma_{2}^{2}} \sum_{l=1}^{2}(-1)^{l+1} \mu_{l m}^{2}\left(H_{0}^{(1)}\left(\mu_{l m} r\right)+H_{2}^{(1)}\left(\mu_{l m} r\right) \cos 2 \alpha\right)+H_{0}^{(1)}\left(\mu_{2 m} r\right), \\
& g_{3 m}^{(2)}=-g_{2 m}^{(3)}=-\frac{\lambda_{m}}{\gamma_{2}^{2}} \sum_{l=1}^{2}(-1)^{l+1} \mu_{l m} H_{1}^{(1)}\left(\mu_{l m} r\right) \sin \alpha, \\
& g_{3 m}^{(3)}=\frac{\lambda_{m}^{2}}{\gamma_{2}^{2}} H(r)+H_{0}^{(1)}\left(\mu_{2 m} r\right) .
\end{aligned}
$$

\section{B The Kernels of integral equations}

$$
\begin{aligned}
K_{11} & =\left[\frac{1}{2(1-v)} \mu_{1 m} H_{1}^{(1)}\left(\mu_{1 m} r_{0}\right)-\lambda_{m}^{2} g_{1 m}+\left\{g_{4 m}+2 \mu_{2 m} H_{1}^{(1)}\left(\mu_{2 m} r_{0}\right)\right\} \mathrm{e}^{2 \mathrm{i}\left(\alpha_{0}-\psi_{0}\right)}\right] \mathrm{e}^{\mathrm{i}\left(\psi-\alpha_{0}\right)}, \\
K_{12} & =\left[\frac{1}{2(1-v)} \mu_{1 m} H_{1}^{(1)}\left(\mu_{1 m} r_{0}\right)-\lambda_{m}^{2} g_{1 m}-g_{3 m} \mathrm{e}^{2 \mathrm{i}\left(\alpha_{0}-\psi_{0}\right)}\right] \mathrm{e}^{\mathrm{i}\left(\alpha_{0}-\psi\right)}, \\
K_{13} & =\lambda_{m}\left[\frac{1}{2(1-v)} H_{0}^{(1)}\left(\mu_{1 m} r_{0}\right)-H_{0}^{(1)}\left(\mu_{2 m} r_{0}\right)-\frac{\lambda_{m}^{2}}{\gamma_{2}^{2}} H\left(r_{0}\right)-g_{2 m} \mathrm{e}^{2 \mathrm{i}\left(\alpha_{0}-\psi_{0}\right)}\right], \\
K_{31} & =2 \lambda_{m}\left[g_{2 m} \mathrm{e}^{2 \mathrm{i}\left(\psi_{0}-\alpha_{0}\right)} g_{0 m}-H_{0}^{(1)}\left(\mu_{2 m} r_{0}\right)\right] \mathrm{e}^{\mathrm{i}\left(\psi-\psi_{0}\right)}, \\
K_{33} & =2\left[2 \lambda_{m}^{2} g_{1 m}+\mu_{2 m} H_{1}^{(1)}\left(\mu_{2 m} r_{0}\right)\right] \cos \left(\alpha_{0}-\psi_{0}\right), \\
\zeta_{0} \in \Gamma & =\cup \Gamma_{\nu}, \quad \psi_{0}=\psi\left(\zeta_{0}\right), \quad \zeta-\zeta_{0}=r_{0} e^{i \alpha_{0}},
\end{aligned}
$$




$$
\begin{aligned}
& g_{0 m}=\frac{1}{\gamma_{2}^{2}} \sum_{l=1}^{2}(-1)^{l+1} \mu_{l m}^{2} H_{0}^{(1)}\left(\mu_{l m} r_{0}\right), \quad g_{j m}=\frac{1}{\gamma_{2}^{2}} \sum_{l=1}^{2}(-1)^{l+1} \mu_{l m}^{j} H_{j}^{(1)}\left(\mu_{l m} r_{0}\right), \quad j=1,2,3, \\
& g_{4 m}=\frac{1}{\gamma_{2}^{2}} \sum_{l=1}^{2}(-1)^{l+1} \mu_{l m}^{3} H_{1}^{(1)}\left(\mu_{l m} r_{0}\right) .
\end{aligned}
$$

The kernels $K_{22}, K_{21}, K_{23}, K_{32}$ are obtained from $K_{11}, K_{21}, K_{13}, K_{31}$ by substituting $\alpha_{0}, \psi_{0}, \psi$ for $-\alpha_{0}$, $-\psi_{0},-\psi$, respectively.

\section{The numerical scheme for the solution of the system of integral equations}

To solve the system of integral equations, we parametrized the contour of the opening as $\zeta=\zeta(\beta)$, where $\beta$ is the real parameter $0 \leq \beta<2 \pi$. On the contour, two systems of points are introduced:

The points $\beta_{k}=\frac{\pi(2 k-1)}{N} k=\overline{1, N}$ of interpolation,

The points $\beta_{0 l}=\frac{2 \pi(l-1)}{N} l=\overline{1, N}$ of collocation.

Here, $N$ is the number of partition points. It is noteworthy to mention that because of the specific features of the used interpolation formula, $N$ is odd.

The integrals were represented in the form of sums with the aid of the following quadrature formula:

$$
\int y(\zeta) K\left(\zeta, \zeta_{0}\right) \mathrm{d} S=\frac{2 \pi}{N} \sum_{k=1}^{N} y_{k} K\left(\zeta_{k}, \zeta_{0}\right) \sqrt{\left(\operatorname{Re} \zeta_{k}^{\prime}\right)^{2}+\left(\operatorname{Im} \zeta_{k}^{\prime}\right)^{2}},
$$

where $\zeta_{k}=\zeta\left(\beta_{k}\right), y_{k}=y\left(\zeta\left(\beta_{k}\right)\right)=y\left(\beta_{k}\right)$.

Extra-integral addends at collocation points are expressed in terms of magnitude $y_{k}$, with the aid of the interpolation formula [77-79]

$$
y_{0 l}=\frac{1}{N} \sum_{k=1}^{N}(-1)^{k+l} \frac{y_{k}}{\sin \left(\left(\beta_{k}-\beta_{0 l}\right) / 2\right)}
$$

where $y_{0 l}=y\left(\zeta\left(\beta_{0 l}\right)\right)=y\left(\beta_{0 l}\right)$.

With the aid of the formulas $(\mathrm{C} 1),(\mathrm{C} 2)$, the integral equations (18) are reduced to a system of linear algebraic equations.

\section{References}

1. Achenbach, J.D.: Wave Propagation in elastic Solids. North-Holland, Amsterdam (1973)

2. Achenbach, J.D.: Transient shear waves in two joint elastic quarter spaces. Int. Appl. Mech. 36, 491-496 (1969)

3. Achenbach, J.D.: Shear waves in an elastic wedge. Int. J. Solids Struct. 6, 379-388 (1970)

4. Bardzokas, D.I., Zobnin, A.I.: Mathematical modelling of Physical processes in composite materials of periodical structure. Moscow Edit. URSS (2003)

5. Bardzokas, D.I., Zobnin, A.I., Senik, N.A., Filshtisnky, M.L.: Mathematical modelling in the problems of the mechanics of coupled fields. Moscow Edit. URSS (2006)

6. Bardzokas, D.I., Filshtinsky, M.L., Filshtinsky, L.A.: Mathematical Methods in Electro-magneto-elasticity. Springer, Berlin (2007)

7. Bardzokas, D.I., Kudryartsev, B.A., Senik, N.A.: Wave propagation in electromagnetoelastic media. Moscow Edit. URSS (2003)

8. Brock, L.M.: Two basic problems of plane crack extension: a unified treatment. Int. J. Eng. Sci. 15, 527-536 (1977)

9. Brock, L.M.: Sliding and indentation by a rigid half-wedge with friction and displacement coupling effects. Int. J. Eng. Sci. 19, 33-40 (1981)

10. Gaginard, L.: Reflection and Refraction of Progressive Seismic Waves in Layered Waves. McGraw-Hill, NY (1957)

11. Freund, L.B.: Dynamic Fracture Mechanics. Cambridge University Press, London (1989)

12. Freund, L.B., Achenbach, J.D.: Diffraction of a plane pulse by a closed crack at the interface of elastic solids. Z. Angew. Math. Mech. 48, 173-185 (2006)

13. Freund, L.B.: The initial wave emitted by a suddenly extending crack in an elastic solid. J. Appl. Mech. 39, 601-602 (1972)

14. Freund, L.B.: Crack propagation in an elastic solid subjected to general loading. III. Stress wave loading. J. Mech. Phys. Solids 21, 47-61 (1973) 
15. Freund, L.B.: The response of an elastic solid to non-uniformly moving surface loads. J. Appl. Mech. 40, 699-704 (1973)

16. Georgiadis, H.G., Barber, J.R.: Steady-state transonic motion of a line load over an elastic half-space. The corrected Cole/Huth solution. J. Appl. Mech. Trans. ASME 60, 772-774 (1993)

17. Georgiadis, H.G., Barber, J.R.: On the super-Rayleigh/subseismic elastodynamic indentation problem. J. Elast. 31, 141-161 (1993)

18. Georgiadis, H.G., Lykotrafitis, G.: A method based on the Radon transform for three-dimensional elastodynamic problems of moving loads. J. Elast. 65, 87-129 (2002)

19. Gurtin, M.E., Stenberg, E.: A note on uniqueness in classical elastodynamics. Quart. Appl. Math. 19, 169-171 (1961)

20. Gurtin, M.E., Toupin, R.A.: A uniqueness theorem for the displacement boundary value problem of linear elastodynamics. Quart. Appl. Math. 23, 79-81 (1965)

21. Graff, K.F.: Wave Motion in Elastic Solids. Ohio State University Press, USA (1975)

22. Hudson, J.A.: The Excitation and Propagation of Elastic Waves. Cambridge University Press, London (1980)

23. Harris, J.G.: Linear Elastic Waves. Cambridge University Press, London (2004)

24. DeHoop, A.T.: A modification of Canginard's method for solving the seismic pulse problem. Appl. Sci. Res. B 8, 349$356(1960)$

25. Ewing, W.M., Jardetzky, W.S., Press, F.: Elastic Waves in Layered Media. McGraw-Hill, NY (1957)

26. Eringen, A.C., Suhubi, E.S.: Elastodynamics. Finite Motions, vol. 1. Academic Press, NY (1975)

27. Eringen, A.C., Suhubi, E.S.: Elastodynamics. Linear Theory, vol. 2. Academic Press, NY (1975)

28. Kolsky, H.: Stress waves in solids. Oxford University Press (Clarendon), London (1953)

29. Jupradge, V.D.: Dynamical problems in elasticity. In: Sneddon, I. N., Hill, R. (eds) Progress in Solid Mechanics, vol. III. North-Holland Publications, Amsterdam (1973)

30. Miklowitz, J.: The Theory of Elastic Waves and Wave Guides. North-Holland, NY (1978)

31. Pao, Y.H., Mow, C.C.: Diffraction of Elastic Waves and Dynamic Stress Concentrations. Crane, Russak and Co., NY (1973)

32. Poruchikov, V.B.: Methods of the Classical Theory of Elastodynamics. Springer, Berlin (1993)

33. Lamb, H.: On the propagation of tremors over the surface of separation of two solids. Proc. Roy. Soc., London Ser. A 203, $1-42(1904)$

34. Royer, D., Dieulesaint, E.: Elastic waves in solids. I. Free and guided propagation. Springer, Berlin (1996)

35. Stoneley, R.: Elastic waves at the surface of separation of two solids. Proc. Roy. Soc. London Ser. A 106, 416-428 (1924)

36. Lurie, A.I.: On the theory of thick plates. Appl. Math. Mech. 6, 151-167 (1942)

37. Prokopov, V.K.: Application of the symbolic method to the derivation of the equations of the theory of plates. J. Appl. Math. Mech. 29, 1064-1083 (1965)

38. Aksentyan, O.K., Vorovich, I.I.: Stress state of a thin plate. Appl. Math. Mech. 27, 1057-1074 (1963)

39. Vorovich, I.I., Malkina, O.S.: The stress state of thick layers. Appl. Math. Mech. 31, 230-241 (1967)

40. Vorovich, I.I.: Certain stress concentration problems. Appl. Math. Mech. 2, 45-53 (1968)

41. Kosmodamianskii, A.S.: Three-dimensional problems of the theory of elasticity for multi-connected plates. Appl. Mech. 19, 3-21 (1983)

42. Altuhov, E.V.: The stress state of thick plates under homogeneous boundary conditions of mixed type on the faces. J. Math. Sci. 76, 2339-2342 (1995)

43. Altuhov, E.V., Misovshii, V.V., Panchenko, Y.V.: Three-dimensional problems of steady vibrations of isotropic plates. J. Math. Sci. 86, 3095-3098 (1997)

44. Altuhov, E.V., Goltsev, A.S., Hiznyak, V.K.: Stress state of isotropic layers with crack. Int. Appl. Mech. 33, 39-46 (1997)

45. Bodnya, Y.N., Shaldyrvan, V.A.: Elastic deformation of thick plates with sliding restraint of the end faces. J. Math. Sci. 57, 2835-2840 (1991)

46. Karapetian, E., Kachanov, M.: Green's functions for the isotropic or transversely isotropic space containing a circular crack. Acta Mech. 126, 169-187 (1998)

47. Bulanov, G.S.: Multisided tension of thick partial homogeneous plates. J. Theor. Appl. Mech. 8, 19-23 (1977)

48. Zhirov, V.E.: Electro-elastic equilibrium of a piezoceramic plate. Appl. Math. Mech. 41, 1114-1121 (1977)

49. Fridman, L.I.: Dynamic problem of the theory of elasticity for bodies of canonical form. Int. Appl. Mech. 23, 1195-1201 (1987)

50. Ulitko, A.F.: The method of vector eigenfunctions in three-dimensional problems of elasticity theory. Int. Appl. Mech. 3, $1-7$ (1967)

51. Grinchenko, V.T., Ulitko, A.F.: Exact solution of the Kirsch problem. Int. Appl. Mech. 6, 455-461 (1970)

52. Kupradze, V.D., Gegelia, T.G., Bagheleishvili, H.O., Burchuladze, T.V.: Three-dimensional problems of the mathematical theory of elasticity and thermo-elasticity. North-Holland, NY (1979)

53. Kit, G.S., Hai, M.V.: The method of potential in three-dimensional problems of thermo-elasticity with cracks. Naukova Dumka, Kiev (In Russian) (1989)

54. Parton, V.Z., Perlin, V.I.: Integral Equations in Elasticity. MIR, Moscow (1982)

55. Voreshko, P.P.: Effective construction of integral equations of potential theory for basic boundary problems of the theory of elasticity. Strength Mater. 5, 83-90 (1996)

56. Tranter, C.J.: Integral Transforms in Mathematical Physics. Wiley, NY (1956)

57. Stenberg, E.: Three-dimensional stress concentrations in the theory of elasticity. Appl. Mech. Rev. 1, 1-4 (1958)

58. Landau, L.D., Lifshitz, E.M.: Theory of Elasticity. Pergamon, NY (1986)

59. Alexandrov, A.Y., Soloviev, Y.I.: Three-dimensional problems of theory of elasticity. Nauka, Moscow (In Russian) (1978)

60. Shapiro, G.S.: On the stress distribution in an unconstrained layer. Appl. Math. Mech. 8, 167-168 (1944)

61. Wang, W., Shi, M.X.: Thick plate theory based on general solutions of elasticity. Acta Mech. 123, 27-36 (1997)

62. Filshtinskii, L.A.: Periodic solutions of the theory of elasticity and electro-elasticity for cylinders in R3. J. Theor. Appl. Mech. 21, 13-20 (1990)

63. Filshtinskii, L.A., Kovalev, Y.D.: Bending of a layer with through tunnel cuts and free-sliding end faces. Mater. Sci. 36, 570-574 (2000) 
64. Grigolyuk, E.I., Filshtinskii, L.A.: Bending of an elastic plate weakened by a doubly periodic system of circular holes. Mech. Comp. Mater. 4, 1-3 (1968)

65. Grigoluk, E.I., Kovalev, Y.D., Filshtinskii, L.A.: Mixed oblique-symmetric problem of the theory of elasticity for weakened from tunnel cracks layers. Rep. Univ. North-Caucasus 3, 46-47 (in Russian) (2000)

66. Filshtinskii, L.A., Kovalev, Yu.D.: Mixed antisymmetric problem of an elastic layer weakened by through cavities. Mater. Sci. 37, 833-837 (2001)

67. Filshtinskii, L.A., Oleinik, V.M.: Boundary value problems of electroelasticity for layers weakened from tunnel cracks. Int. Appl. Mech. 27, 21-26 (1991)

68. Filshtinskii, L.A., Oleinik, V.M.: Three-dimensional problems of electroelasticity for layers weakened from tunnel cracks. Phys. Chem. Mech. Mater. 27, 66-72 (1991)

69. Filshtinskii, L.A.: Tension of a layer weakened from tunnel cracks. Appl. Math. Mech. 59, 827-835 (1995)

70. Filshtinskii, L.A.: Fundamental solutions for the equations of electroelasticity for piezoceramic layers in R3. Mech. Comp. Mater. 31, 377-388 (2001)

71. Filshtinskii, L.A.: Periodic in time fundamental solutions for the equations of thermoelasticity for anisotropic layers in R3. Math. Methods Phys. Mech. 46, 147-154 (2003)

72. Filshtinskii, L.A., Kovalev, Yu.D., Ventsel, E.S.: Solution of the elastic boundary value problems for a layer with tunnel stress raisers. Int. J. Solids Struct. 39, 6385-6402 (2002)

73. Filshtinksii, L.A., Sirenko, Y.V.: Homogeneous solutions of the Neumann problem for a composite layer in R3. Curr. Problems Continuum Mech. 1, 200-2005 (2005)

74. Filshtinskii, L.A., Shramko, L.V., Shramko, Y.V.: Fundamental solutions for piezoceramic layers in R3-symmetric case, mixed boundary conditions. Curr. Problems Continuum Mech. 2, 199-203 (2003)

75. Filshtinskii, L.A.: Fundamental solutions of electroelasticity equations for a piezoceramic layer in R3. Mech. Comp. Mater. 37, 234-237 (2001)

76. Nazarenko, A.M., Filshtinskii, L.A.: Interaction of elastic waves with a curvilinear crack in a half-plane. J. Math. Sci. 57, 2887-2891 (1991)

77. Tikhonov, A.N., Samarskii, A.A.: Equations of Mathematical Physics. Dover Publications, NY (1990)

78. Theocaris, P.S.: Numerical solution of singular integral equations: methods. J. Eng. Mech. Div. ASCE 107, 733-752 (1981)

79. Theocaris, P.S.: Numerical solution of singular integral equations: applications. J. Eng. Mech. Div. ASCE 107, 753-771 (1981) 